\title{
Comparative Transcriptomic Analysis of Differentially Expressed Transcripts Associated with Flowering Time of Loquat (Eriobotya japonica Lindl.)
}

\author{
Haishan An ${ }^{1,2,+}{ }^{\circ}$, Shuang Jiang ${ }^{1,2,+}$, Jiaying Zhang ${ }^{1,2}$, Fangjie $X u^{1,2}$ and Xueying Zhang ${ }^{1,2, *}$ \\ 1 Forestry and Pomology Research Institute, Shanghai Academy of Agricultural Sciences, No. 1000 Jinqi Road, \\ Fengxian District, Shanghai 201403, China; anhaishan530@163.com (H.A.); jiangshuang@saas.sh.cn (S.J.); \\ zhangjiaying@saas.sh.cn (J.Z.); xufangiie@saas.sh.cn (F.X.) \\ 2 Shanghai Key Lab of Protected Horticultural Technology, No. 1000 Jinqi Road, Fengxian District, \\ Shanghai 201403, China \\ * Correspondence: zhangxueying@saas.sh.cn \\ + These authors contributed equally to this work.
}

Citation: An, H.; Jiang, S.; Zhang, J.; $\mathrm{Xu}, \mathrm{F}$; Z Zhang, X. Comparative Transcriptomic Analysis of Differentially Expressed Transcripts Associated with Flowering Time of Loquat (Eriobotya japonica Lindl.). Horticulturae 2021, 7, 171. https:// doi.org/10.3390/horticulturae7070171

Academic Editor: Nakao Kubo

Received: 8 May 2021

Accepted: 25 June 2021

Published: 1 July 2021

Publisher's Note: MDPI stays neutral with regard to jurisdictional claims in published maps and institutional affiliations.

Copyright: () 2021 by the authors. Licensee MDPI, Basel, Switzerland. This article is an open access article distributed under the terms and conditions of the Creative Commons Attribution (CC BY) license (https:// creativecommons.org/licenses/by/ $4.0 /)$.

\begin{abstract}
Flowering is an important phenophase of plant species, however, knowledge about the regulatory mechanism controlling flowering cues in loquat is limited. To identify candidate genes regulating flowering time in loquat, we used RNA-Seq technology to conduct a comparative transcriptome analysis of differentiating apical buds collected from the early-flowering variety 'Baiyu' and the late-flowering variety 'Huoju'. A total of 28,842 differentially expressed transcripts (DETs) were identified. Of these, $42 \mathrm{DETs}$ controlled flowering time while 17 other DETs were associated with the ABA signaling pathway. Compared with those in 'Huoju', EjFT, EjFY, EjFLK, and EjCAL1like were significantly upregulated in 'Baiyu'. Moreover, transcripts of the ABA 8'-hydroxylases (EjABH2, EjABH4, and EjABH4-like2), the ABA receptors (EjPYL4/8), and the bZIP transcription factor EjABI5-like were upregulated in 'Baiyu' compared with 'Huoju'. Hence, they might regulate loquat flowering time. There was no significant difference between 'Baiyu' and 'Huoju' in terms of IAA content. However, the ABA content was about ten-fold higher in the apical buds of 'Baiyu' than in those of 'Huoju'. The ABA:IAA ratio sharply rose and attained a peak during bud differentiation. Thus, ABA is vital in regulating floral bud formation in loquat. The results of the present study help clarify gene transcription during loquat flowering.
\end{abstract}

Keywords: differentially expressed transcript; Eriobotya japonica Lindl.; flowering time; transcriptomic analysis

\section{Introduction}

Flowering is the onset of plant reproduction and an important phenophase. Understanding the mechanisms that control flowering events is essential for efficient fruit reproduction [1]. Flowering has evolved to maximize outcrossing and mitigate the damaging effects of frost. It involves elegant mechanisms that integrate complex signals including photoperiod and temperature [2,3]. Flower bud differentiation and, by extension, flowering itself, are influenced by sophisticated regulatory networks [4-7]. Several major pathways in Arabidopsis include floral integrator genes that govern flowering time. Photoperiod as well as circadian, ambient temperature, vernalization, autonomous, gibberellin, and aging pathways are implicated [8]. FLOWERING LOCUS T (FT) is a crucial mobile signal [9]. FT and FT-like play pivotal roles in the flowering of fruit trees in the Rosaceae family such as apple [10,11], pear [12], peach [13], and loquat [14-16]. Flowering time is affected by environmental conditions and phytohormones. Previous studies have demonstrated phytohormone crosstalk in flower induction $[17,18]$. ABA is generally considered a flowering suppressor [19]. In loquat, however, ABA plays a positive role in flower bud 
formation $[16,20]$. The ABA content dramatically rises in loquat apical buds and strongly influences loquat flower bud induction and differentiation [20]. To date, however, little is known about the mechanism by which ABA promotes loquat bud differentiation or the ABA-associated regulatory pathway that controls loquat flowering time.

Loquat (Eriobotrya japonica Lindl.) (Rosaceae) is a subtropical evergreen fruit tree native to China. It blooms in autumn and forms terminal panicles in early winter [14,21]. Loquat floral buds are usually derived from vegetative buds on shoots produced mainly during summer [22]. The loquat flower bud may persist for several months. Bud initiation may require special environmental conditions such as high temperature and moderate drought stress [23]. In Shanghai, China, loquat flower bud differentiation occurs between July and September followed by blooming from early October to January of the following year. However, the mechanisms regulating loquat bud differentiation and flowering time are poorly understood. RNA-Seq technology comprehensively identifies transcripts and facilitates transcriptome analysis. It enables rapid gene discovery and creates more sensitive and accurate transcriptome profiles than other techniques [24]. RNA-Seq recently revealed candidate genes regulating specific biological processes in triploid loquat such as the gibberellin-induced fruit setting pathway [24], fruit development and ripening [25], and low-temperature stress response [26,27]. However, neither transcript information nor gene expression profiling during loquat flower bud differentiation has been determined. Hence, the application of RNA-Seq to identify candidate genes associated with flowering time in loquat would help elucidate the mechanism by which this process is controlled.

In the present study, a comparative transcriptome analysis was conducted to reveal the molecular mechanism regulating flowering time in loquat. Differentiating flower buds were collected from the early-flowering variety 'Baiyu' and the late-flowering variety 'Huoju'. Differentially expressed transcripts (DETs) were explored to identify candidate genes involved in the regulation of flowering in loquat.

\section{Materials and Methods}

\subsection{Plant Materials}

Two loquat (Eriobotrya japonica Lindl.) varieties with distinct flowering traits were used in this study. 'Baiyu' is an early-flowering cultivar while 'Huoju' is a late-flowering variety. Both were planted at the Jinshan Fruit Tree Experimental Station of the Shanghai Academy of Agricultural Sciences, Shanghai, China $\left(30^{\circ} 47^{\prime} 27^{\prime \prime} ; 121^{\circ} 8^{\prime} 6^{\prime \prime}\right)$. During flower bud differentiation (early July to late August in Shanghai), about 30 apical buds were randomly picked at 7-d intervals between 13 July 2017 and 24 August 2017. All samples were immediately frozen in liquid nitrogen and stored at $-80^{\circ} \mathrm{C}$ before being analyzed.

\subsection{Endogenous Phytohormone Determination by LC-MS}

About $0.5 \mathrm{~g}$ of fresh apical bud was ground in liquid nitrogen and homogenized in $5 \mathrm{~mL}$ of ethyl acetate. The suspension was vortexed for $1 \mathrm{~min}$ and stored in the dark at $4{ }^{\circ} \mathrm{C}$ for $12 \mathrm{~h}$. The mixture was centrifuged at $10,000 \times g$ and $4{ }^{\circ} \mathrm{C}$ for $10 \mathrm{~min}$. The supernatant was collected and the residue was extracted twice with $3 \mathrm{~mL}$ ethyl acetate at $10,000 \times g$ and $4{ }^{\circ} \mathrm{C}$. The solution was vacuum-concentrated to dryness at $30^{\circ} \mathrm{C}$ and the residue was dissolved in $250 \mathrm{~mL}$ chromatographic methanol. The solution was then passed through a $0.22-\mu \mathrm{m}$ membrane filter and a $10 \mu \mathrm{L}$ aliquot was injected into a liquid chromatograph-mass spectrometer (LC-MS). Changes in the levels of endogenous phytohormones including abscisic acid (ABA) and indoleacetic acid (IAA) at each sampling period were analyzed according to the methods of Niu et al. (2014) [28]. The mobile phase consisted of methanol and $0.5 \%(v / v)$ acetic acid dissolved in redistilled water. The standards were IAA (I2886) and ABA (A1094) (Sigma-Aldrich Corp., St. Louis, MO, USA). Calibration curves were plotted using $0 \mathrm{ng} / \mathrm{mL}, 2.5 \mathrm{ng} / \mathrm{mL}, 5.0 \mathrm{ng} / \mathrm{mL}, 10 \mathrm{ng} / \mathrm{mL}, 12.5 \mathrm{ng} / \mathrm{mL}, 25 \mathrm{ng} / \mathrm{mL}, 50 \mathrm{ng} / \mathrm{mL}$, and $100 \mathrm{ng} / \mathrm{mL}$ of each standard. Linear regressions of the calibration curves are shown in Table S1. 


\subsection{Total RNA Extraction and RNA-Seq}

Total RNA was extracted with a mirVana miRNA Isolation Kit (Thermo Fisher Scientific, Waltham, MA, USA) according to the manufacturer's protocol. RNA integrity was validated with an Agilent 2100 Bioanalyzer (Agilent Technologies, Santa Clara, CA, USA). RNA-Seq libraries were constructed with a TruSeq Stranded mRNA LTSample Prep Kit (Illumina, San Diego, CA, USA) and applied to an Illumina HiSeq X Ten sequencing platform for RNA-Seq analysis by Shanghai OE Biotech. Co. Ltd. (Shanghai, China).

\subsection{Quality Control and de Novo Assembly}

Raw data (reads) were processed with Trimmomatic [29]. Low-quality reads and those containing poly- $N$ were removed to obtain clean reads. Adaptor and low-quality sequences were removed and the clean reads were assembled into expressed sequence tag clusters (contigs). They were then assembled de novo into transcripts by the paired-end method using Trinity v. trinityrnaseq_r20131110 [30]. The longest transcript was selected as a unigene based on sequence similarity and length, and used in the subsequent analysis.

\subsection{Functional Annotation}

Unigene function was annotated by aligning the unigenes with the databases NCBI non-redundant (NR), SwissProt, and Clusters of Orthologous Groups for Eukaryotic Complete Genomes (KOG). For this purpose, Blastx was used at a threshold $E$-value of $10^{-5}$ [31] Proteins with the highest numbers of hits to the unigenes were used to assign functional annotations to them. Gene ontology (GO) classification was performed by the mapping relationship between SwissProt and the GO terms. The unigenes were mapped to the Kyoto Encyclopedia of Genes and Genomes (KEGG) database to annotate their potential metabolic pathways [32].

\subsection{Identification of Differentially Expressed Transcripts (DETs) Involved in Flowering Time}

Fragments per kilobase per million mapped reads (FPKM) and read count values for each unigene were calculated with bowtie2 and eXpress [33-35]. Differentially expressed transcripts (DETs) were identified with the DESeq (2012) functions SizeFactors and nbinomTest [36]. $p<0.05$ and FoldChange $>2$ were set as the thresholds for significant differential expression. A hierarchical cluster analysis of the DETs was performed to explore transcript expression patterns. GO and KEGG pathway enrichment analyses of the DETs were performed on the hypergeometric distribution.

\subsection{Gene Expression Analysis by qRT-PCR}

Total RNA was extracted from the loquat apical buds collected at each sampling date. First-strand cDNA was synthesized from $1 \mathrm{~g}$ total RNA using the PrimerScript RT Reagent Kit with gDNA Eraser (RR047; TaKaRa Bio Inc., Shiga, Japan), diluted tenfold with $\mathrm{ddH}_{2} \mathrm{O}$, and used as templates for qRT-PCR. The qRT-PCR mixture $(10 \mu \mathrm{L})$ comprised $5 \mu \mathrm{L}$ SYBR Premix ExTaq (RR820; TaKaRa), $0.5 \mu \mathrm{L}$ of each primer $(10 \mu \mathrm{M}), 1 \mu \mathrm{L}$ cDNA, and $2.5 \mu \mathrm{L}$ RNase-free water. The qRT-PCR was performed on a LightCycler480 instrument (Roche Diagnostics, Basel, Switzerland) according to the manufacturer's instructions. The two-step qRT-PCR program was as follows: $95^{\circ} \mathrm{C}$ for $30 \mathrm{~s}$, followed by 40 cycles at $95^{\circ} \mathrm{C}$ for $5 \mathrm{~s}$ and $60{ }^{\circ} \mathrm{C}$ for $20 \mathrm{~s}$ [24]. Template-free controls were included in each run for each primer pair. $\beta$-actin was the internal reference. Degenerate primer sequences for the qRT-PCR are shown in Table S2.

\subsection{Statistical Analysis}

The data were subjected to one-way analysis of variance (ANOVA) in SPSS v. 18.0 (SPSS Inc., Chicago, IL, USA). Differences between treatment means were considered significant at $p \leq 0.05$. Graphics were created with GraphPad Prism v. 6.0 (GraphPad Software Inc., La Jolla, CA, USA). 


\section{Results}

\subsection{Loquat Apical Bud Phytohormone Analyses}

The IAA levels were low in the 'Baiyu' and 'Huoju' apical buds throughout bud differentiation. They did not significantly differ from each other (Figure 1a). The ABA level in 'Baiyu' was about tenfold higher than that in 'Huoju' and reached maxima at the sampling dates of 3 August and 10 August (Figure 1b). The ABA:IAA ratios varied widely between cultivars (Figure 1c). In 'Baiyu', ABA:IAA exhibited a 'down-up-down' trend. It was relatively lower at the earlier stage (13-27 July, i.e., T0-T2), but sharply rose at sampling time (3 August, T3). Hence, this period might have been the key flower bud differentiation stage (Figure 1c). ABA:IAA did not fluctuate in 'Huoju' throughout the experimental period (Figure 1c). The foregoing results indicated that elevated ABA content and ABA:IAA balance might be required for loquat flower bud differentiation.
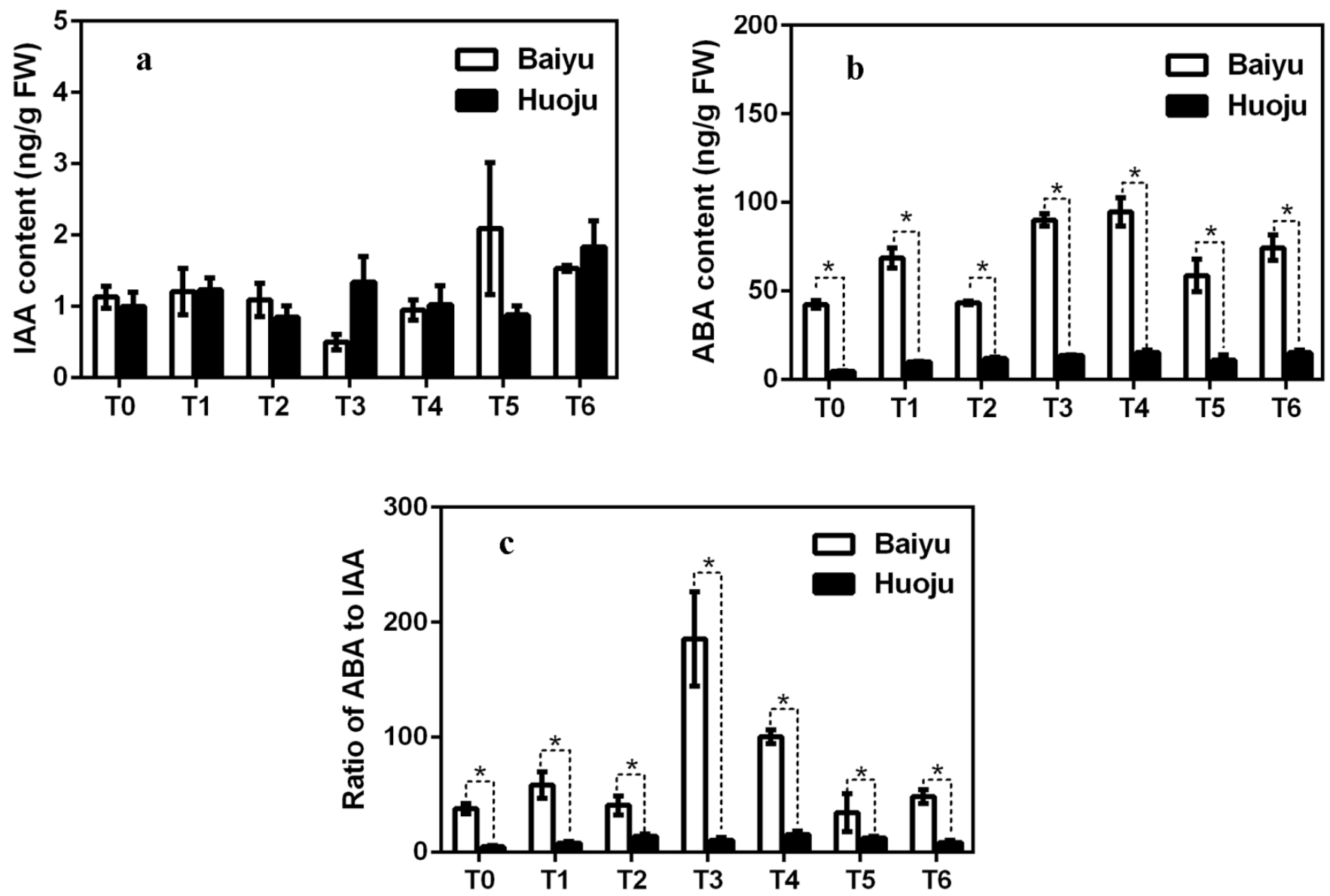

Figure 1. Dynamics of endogenous phytohormone IAA (a), ABA (b) and ratio of ABA to IAA (c) during loquat flower bud differentiation. T0-T6 in horizontal axis indicates sampling dates, i.e., 7-13, 7-19, 7-27, 8-03, 8-10, 8-17, and 8-24, respectively. ${ }^{*}$ indicates significance at $p \leq 0.05$. Bars indicate standard error.

\subsection{Sequence Analysis and Assembly}

To obtain a transcriptome landscape for flower bud differentiation, cDNA samples were extracted from the 'Baiyu' and 'Huoju' apical buds at each sampling date and sequenced on the Illumina HiSeq 4000 platform. Table 1 is an overview of cDNA sequencing. A total of 46.84-49.19 million (M) pairs of 150-bp reads were generated from 'Baiyu' (Table 1). After stringent quality assessment and data cleaning, 40.85-46.91 M clean reads with Q30 (base quality > 30) remained. Their Q30 and GC content ranges were 87.95-93.54\% and $46.92-47.44 \%$, respectively (Table 1 ). For 'Huoju', high-throughput sequencing generated 48.97-49.89 M pairs of 150-bp reads and 46.26-47.56 M clean reads with Q30 were 
generated after stringent quality filtering. Their Q30 and GC content ranges were 93.00 $93.70 \%$ and $46.71-47.23 \%$, respectively (Table 1 ).

Table 1. IIIumina transcriptomes of apical buds during loquat flower bud differentiation.

\begin{tabular}{cccccc}
\hline Cultivar & Sampling Date & Raw Reads & Clean Reads & Q30 (\%) & GC (\%) \\
\hline 'Baiyu' & T0 & $48,991,094$ & $46,581,732$ & 93.30 & 47.44 \\
& T1 & $46,842,090$ & $44,358,410$ & 93.02 & 47.14 \\
& T2 & $49,193,824$ & $46,596,186$ & 93.12 & 47.02 \\
& T3 & $47,387,894$ & $40,851,014$ & 87.95 & 47.36 \\
& T4 & $49,068,496$ & $46,912,890$ & 93.54 & 47.02 \\
& T5 & $48,987,770$ & $46,583,754$ & 93.22 & 47.22 \\
& T6 & $48,997,596$ & $46,632,968$ & 93.14 & 46.92 \\
& T0 & $49,150,924$ & $47,013,524$ & 93.70 & 46.93 \\
& T1 & $49,784,092$ & $47,560,900$ & 93.54 & 46.99 \\
& T2 & $49,889,156$ & $47,546,378$ & 93.35 & 47.13 \\
& T3 & $49,605,682$ & $47,133,928$ & 93.29 & 47.57 \\
& T4 & $48,971,664$ & $46,264,188$ & 93.00 & 47.23 \\
& T5 & $49,348,500$ & $46,926,020$ & 93.17 & 47.14 \\
& T6 & $49,333,148$ & $47,048,570$ & 93.23 & 46.71
\end{tabular}

Note: T0-T6 in horizontal axis indicates sampling dates, i.e., 7-13, 7-19, 7-27, 8-03, 8-10, 8-17, and $8-24$, respectively.

Next-generation short-read sequences were assembled de novo with Trinity (v. trinityrnaseq_r20131110) into 162,080 unigenes with mean size $=1109.34 \mathrm{bp}$ and N50 length $=1625 \mathrm{bp}$ (Table 2). Unigene length was in the range of 301-2000 nt, and the total length was $87,192,678$ nt (Table 2; Figure S1). Distribution of the unigene lengths are shown in Figure S1.

Table 2. Summary statistics of assembled gene sequences.

\begin{tabular}{cccccccc}
\hline Term & $\mathbf{3 0 0} \mathbf{b p}$ & $\mathbf{2} \mathbf{5 0 0} \mathbf{b p}$ & $\mathbf{2} \mathbf{1 0 0 0} \mathbf{b p}$ & Total Number & Total Length (nt) & Average Length & N50 \\
\hline Unigene & 78,599 & 55,189 & 28,292 & 162,080 & $87,192,678$ & 1109.34 & 1625 \\
\hline
\end{tabular}

\subsection{Sequence Annotation}

Several complementary approaches were utilized to annotate the assembled sequences. The sequences were aligned against the NR, Swissport, KEGG, KOG, eggNOG, GO, and Pfam databases using the Diamond program. The threshold $E$-value was $10^{-5}$, and 45,107 NR, 31,795 Swissport, 15,890 KEGG, 23,886 KOG, 38,848 eggNOG, 28,705 GO, and 57 Pfam sequences were identified (Table 3). All unigenes were annotated and matched against loquat genome database (Table S3), and distribution of the top ten species was determined by the Pollution_test based on BLASTx and the NT database. The cutoff $E$-value was $1 \times \mathrm{e}^{-10}$, and coverage was $>80 \%$. About $47.96 \%, 46.53 \%$, and $2.21 \%$ of the unigenes matched sequences from Malus domestica, Pyrus $\times$ bretschneideri, and Eriobotrya japonica, respectively (Figure $\mathrm{S} 2$ ).

Table 3. Summary statistics of unigenes annotated by different databases.

\begin{tabular}{cccc}
\hline Anno_Database & Annotated Number & $\mathbf{3 0 0} \leq$ Length $<\mathbf{1 0 0 0}$ & Length $\geq \mathbf{1 0 0 0}$ \\
\hline NR & $45,107(57.39 \%)$ & $20,034(25.49 \%)$ & $25,073(31.90 \%)$ \\
Swissport & $31,795(40.45 \%)$ & $11,273(14.34 \%)$ & $20,522(26.11 \%)$ \\
KEGG & $15,890(20.22 \%)$ & $6774(8.62 \%)$ & $9116(11.60 \%)$ \\
KOG & $23,886(30.39 \%)$ & $8853(11.26 \%)$ & $15,033(19.13)$ \\
eggNOG & $38,848(49.43 \%)$ & $15,125(19.24 \%)$ & $23,723(30.18 \%)$ \\
GO & $28,705(36.52 \%)$ & $10,314(13.12 \%)$ & $18,391(23.40 \%)$ \\
Pfam & $57(0.07 \%)$ & $50(0.06 \%)$ & $7(0.01 \%)$ \\
\hline
\end{tabular}


GO and KEGG analysis categorized the functions of the predicted loquat genes (Tables S4 and S5). The unigenes $(28,705)$ were classified into three main categories, including 'biological process' $(24,099)$, 'cellular component' $(25,113)$, and 'molecular function' $(24,690)$ (Figure 2). In the 'biological process' category, the unigenes implicated in 'cellular process' and 'metabolic process' predominated. There were also high percentages of unigenes under the categories 'response to stimulus', 'biological regulation', and 'regulation of biological process'. For the 'cellular component' category, many unigenes were categorized under 'cell', 'cell part', and 'organelle'. Under the 'molecular function' category, most of the unigenes were involved in 'binding', 'catalytic activity', and 'transporter activity'. The GO enrichment of whole uingenes in each library is shown in Figures S9-S15.

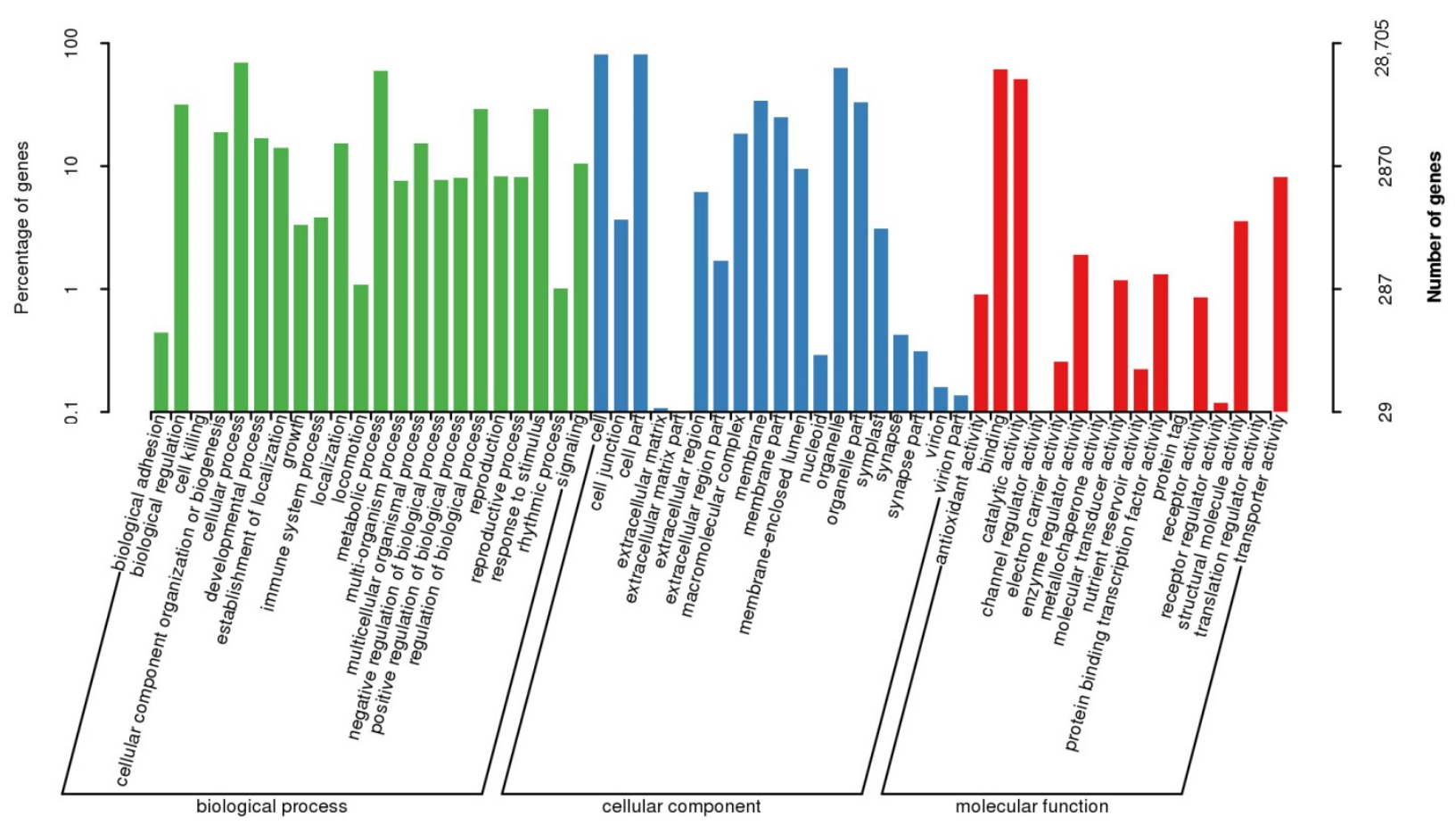

Figure 2. Functional annotation of loquat transcripts based on gene ontology categorization. A total of 28,705 unigenes were classified in the categories 'biological process' $(24,099)$, 'cellular component' $(25,113)$, and 'molecular function' $(24,690)$. Note: The left $x$-axis represents the $\%$ of a specific class of genes in the main category. The right $y$-axis represents the number of genes in each category.

\subsection{Loquat DET Identification}

Seven cDNA libraries (Huoju_713-vs-Baiyu_713, Huoju_719-vs-Baiyu_719, Huoju_727vs-Baiyu_727, Huoju_803-vs-Baiyu_803, Huoju_810-vs-Baiyu_810, Huoju_817-vs-Baiyu_817, and Huoju_824-vs-Baiyu_824) were sequenced, and FPKMs were calculated for all unigenes. Differences in gene expression were evaluated based on $p<0.05$ and FoldChange $>2$. DETs were identified by pairwise comparisons against each library (Figure S3); unigenes obtained by RNA-Seq were matched to gene ID in loquat genomic database (Table S6). A total of 28,842 DETs were detected. Of these, 15,383 were upregulated and 13,459 were downregulated (Table 4). At each stage, 4109 DETs (1885 upregulated and 2224 downregulated) were detected for Huoju_713-vs-Baiyu_713; 4335 DETs (2628 upregulated and 1707 downregulated) were detected for Huoju_719-vs-Baiyu_719; 4327 DETs (2341 upregulated and 1986 downregulated) were detected for Huoju_727-vs-Baiyu_727; 3252 DETs (1339 upregulated and 1913 downregulated) were detected for Huoju_803-vs-Baiyu_803; 4160 DETs (2039 upregulated and 2121 downregulated) were detected for Huoju_810vs-Baiyu_810; 4112 DETs (2420 upregulated and 1692 downregulated) were detected for Huoju_817-vs-Baiyu_817; and 4547 DETs (2731 upregulated and 1816 downregulated) were detected for Huoju_824-vs-Baiyu_824 (Table 4). Most DETs either increased or decreased. 
GO and KEGG enrichment revealed 284 upregulated and 259 downregulated transcripts common to all seven libraries (Figure 3 ).

Table 4. Statistics of upregulated or downregulated unigenes in each library.

\begin{tabular}{cccc}
\hline Item & Upregulated & Downregulated & Total \\
\hline Huoju_713-vs-Baiyu_713 & 1885 & 2224 & 4109 \\
Huoju_719-vs-Baiyu_719 & 2628 & 1707 & 4335 \\
Huoju_727-vs-Baiyu_727 & 2341 & 1986 & 4327 \\
Huoju_803-vs-Baiyu_803 & 1339 & 1913 & 3252 \\
Huoju_810-vs-Baiyu_810 & 2039 & 2121 & 4160 \\
Huoju_817-vs-Baiyu_817 & 2420 & 1692 & 4112 \\
Huoju_824-vs-Baiyu_824 & 2731 & 1816 & 4547 \\
\hline
\end{tabular}

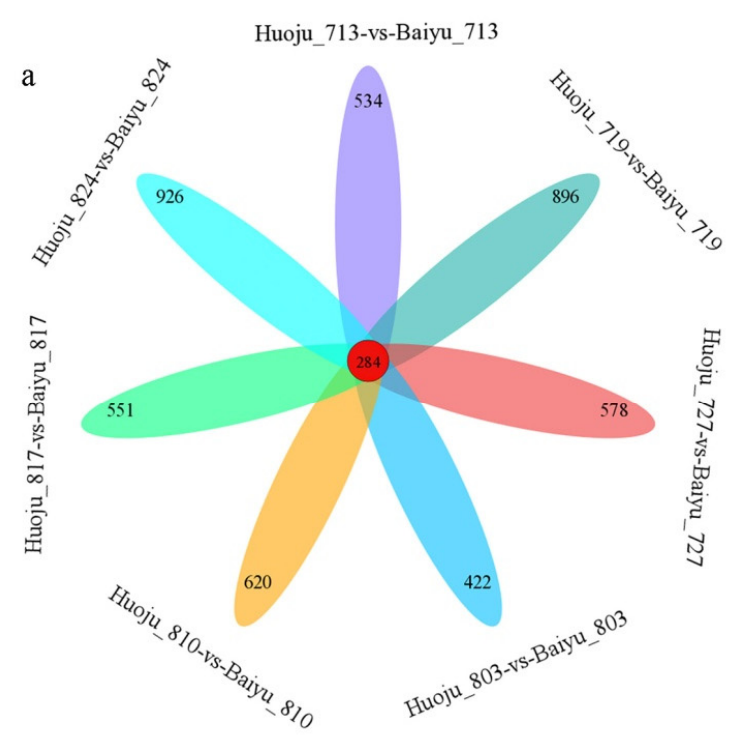

Up-regulated

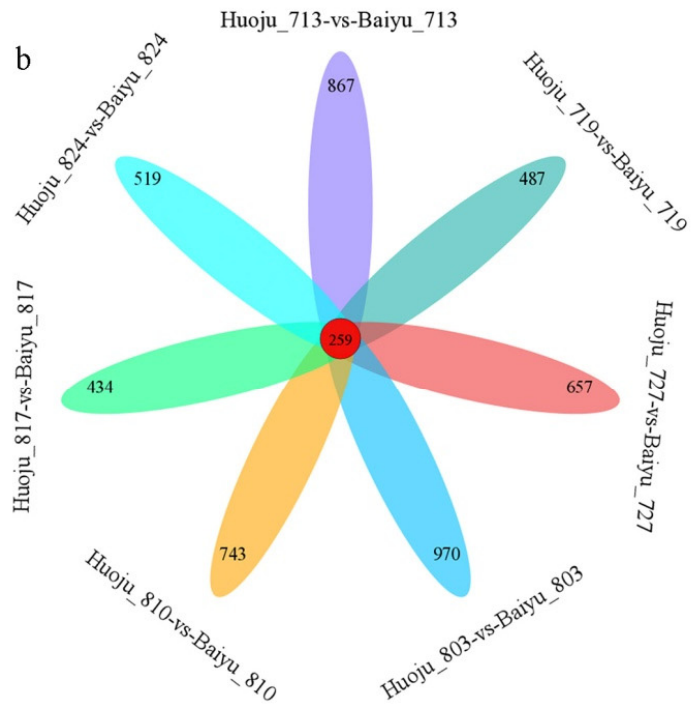

Down-regulated

Figure 3. Venn diagram showing numbers of upregulated (a) and downregulated (b) DETs in each sampling period. Note: Arabic numbers in center indicate the DETs common to seven libraries; Arabic numbers in margin indicate the DETs individually owned in each library, respectively.

\subsection{Identification of DETs Involved in Flowering Time}

We calculated the distributions of the unigenes in the various pathways to identify the DETs controlling flowering time in 'Baiyu' and 'Huoju'. Based on the FPKM values, GO or KEGG enrichment, 15 pathways were enriched. The most representative pathways were flower development, regulation of flower development, and flower morphogenesis. A total of 42 other unigenes were identified, including 5 EARLY FLOWERING-like proteins, 11 flowering time control proteins such as FY-like, FPA, and FCA-like, 12 FLOWERING LOCUS proteins, 8 EMBRYONIC FLOWER-LIKE proteins, and 6 other flowering-associated unigenes (Figure 4, Table S7).

Flowering locus T (EjFT, CL3783Contig2), flowering time control protein FY-like (EjFY, CL21741Contig1), flowering locus K homology domain-like (EjFLK, CL9491Contig1), and truncated transcription factor CAULIFLOWER A-like (EjCAL1, Comp12801_c0_seq1_1) were selected to analyze the relative differences in their expression during 'Baiyu' and 'Huoju' flower bud differentiation. A qRT-PCR evaluated the expression profiles obtained from RNA-Seq data (Figure 5). EjFT, EjFY, EjFLK, and EjCAL1-like were significantly upregulated in the apical buds of 'Baiyu' compared with those of 'Huoju', especially in the early stage of 13-27 July (T0-T2) which was the key period of loquat flower bud differentiation. Hence, these genes play vital roles in flowering time regulation (Figure 5). 
Transcriptome data showed that the EjFY and EjFLK expression levels were higher in

'Baiyu' than 'Huoju' throughout apical bud differentiation (Figure S4b,c).
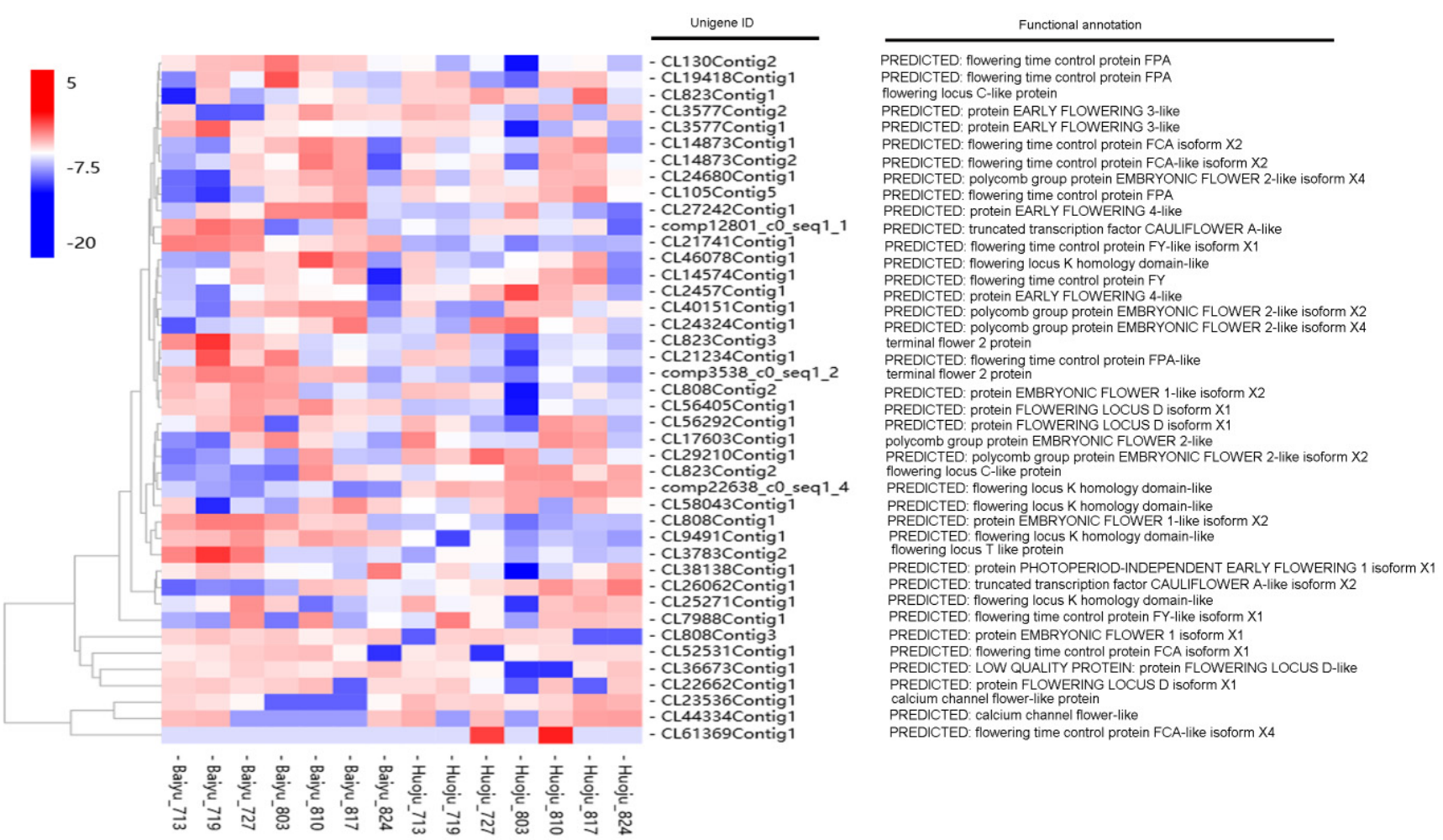

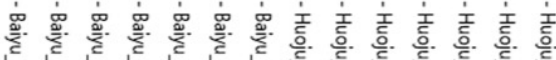

Figure 4. Heatmap of 42 unigenes involved in flowering time regulatory pathways.
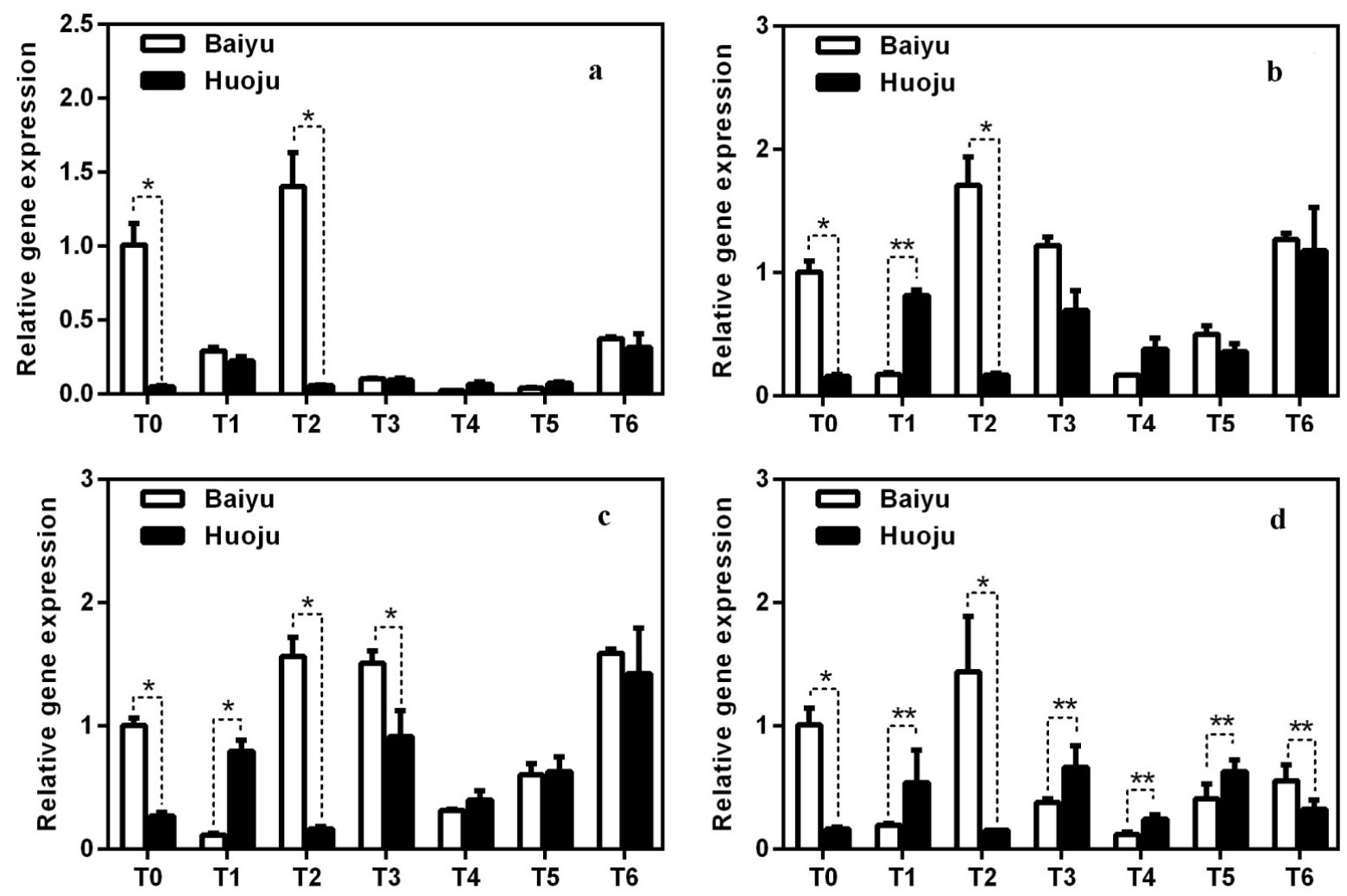

Figure 5. Expression levels of EjFT (a), EjFY (b), EjFLK (c), and EjCAL1-like (d) during loquat bud differentiation. Note: T0-T6 in horizontal axis indicates sampling dates, i.e., 7-13, 7-19, 7-27, 8-03, 8-10, 8-17, and 8-24, respectively; * indicates significance at $p \leq 0.05 ;{ }^{* *}$ indicates significance at $p \leq 0.01$; bars indicate standard error. 


\subsection{Identification of DETs Involved in ABA Signalling}

ABA plays important roles in regulating loquat apical bud differentiation and flowering time. Based on the FPKM values and the GO or KEGG enrichment analyses, we identified DETs involved in ABA signaling. A total of $17 \mathrm{ABA}$ signaling pathways were enriched. The most representative pathways were ABA response, ABA biosynthesis, $\mathrm{ABA}$ transmembrane transporter activity, and others. After GO enrichment at $p=0.05,23$ DETs directly involved in ABA signaling were identified, including $9 \mathrm{ABA} 8^{\prime}$-hydroxylases $($ EjABH), 7 ABA receptors (PYL-likes; EjPYL-likes), and 7 bZIP transcription factors ( $A B-$ SCISIC ACID-INSENSITIVE 5-like proteins; EjABI5-like) (Figure 6, Table S8).

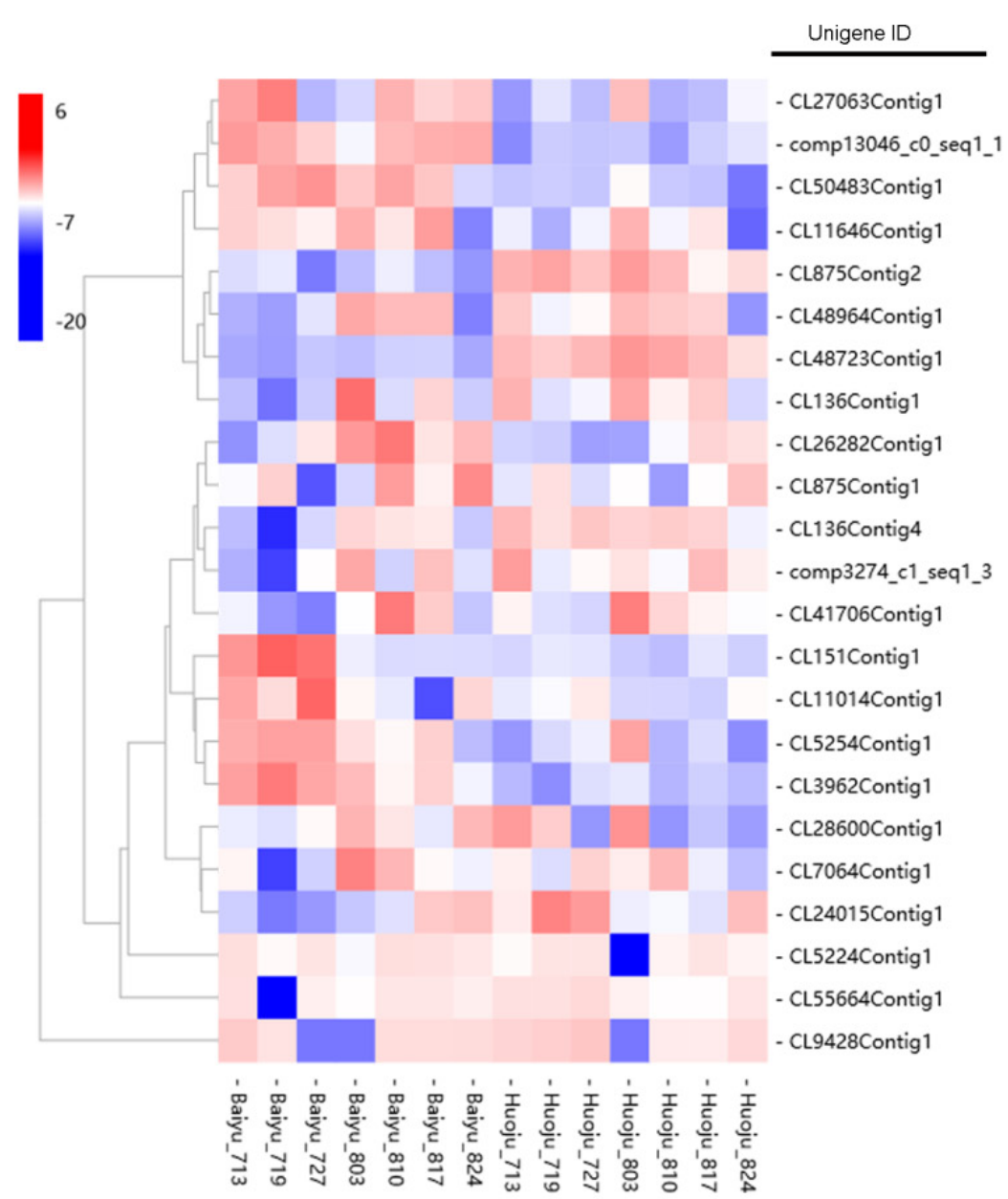

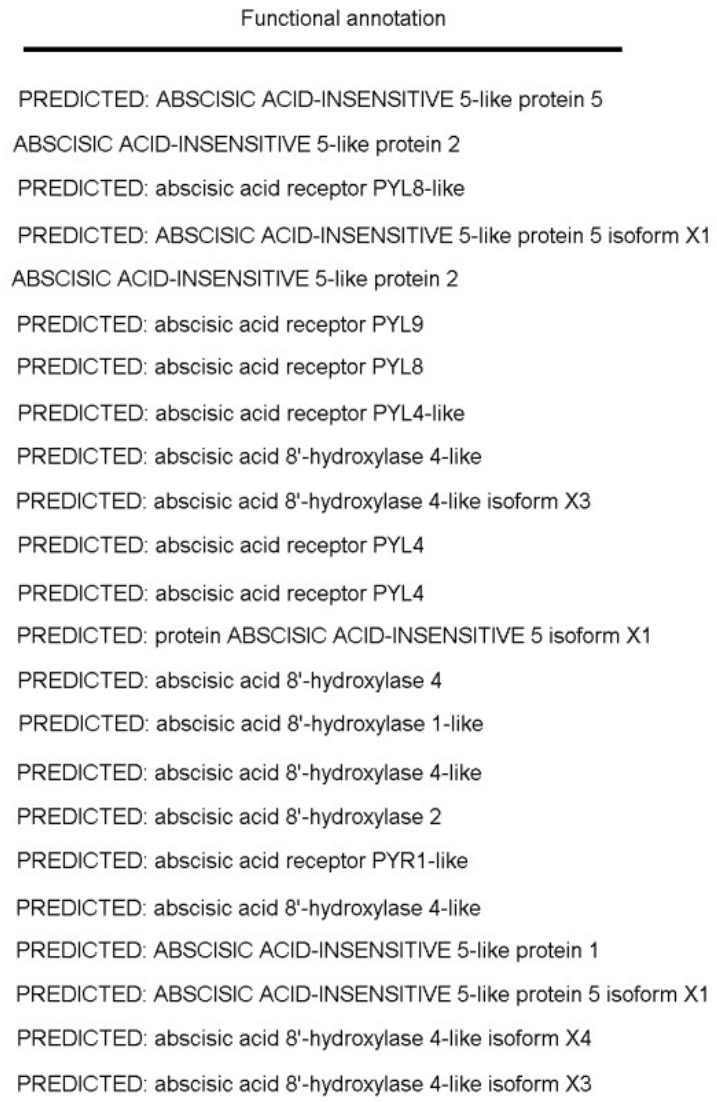

Figure 6. Heatmap of 23 DETs involved in the ABA signaling pathway.

We selected eight DETs associated with the ABA signaling pathway, namely, EjABH2 (CL3962Contig1), EjABH4 (CL151Contig1), EjABH4-like (CL9428Contig1), EjABH4-like2 (CL5254Contig1), EjPYL4 (CL136Contig4), EjPYL4-like (CL136Contig1), EjPYL8 (CL48723Contig1), and EjABI5-like (comp13046_c0_seq1_1). We then determined the relative differences in their expression levels during 'Baiyu' and 'Huoju' flower bud differentiation (Figures 7 and 8). The qRT-PCR evaluated the expression profiles of the foregoing ABA signaling-related DETs, and the output of this analysis showed good agreement with the RNA-Seq data (Figures 7 and 8, Figures S5 and S6). EjABH2, EjABH4, and EjABH4-like2 were significantly upregulated in the apical buds of 'Baiyu' compared with those of 'Huoju', especially at the early stage of 13 July (T0) to 3 August (T3). Hence, these DETs might play vital roles in regulating flowering time. However, EjABH4-like was upregulated in 'Huoju' but downregulated in 'Baiyu' between 13 July (T0) and 27 July (T2). Therefore, EjABH4-like might negatively regulate flowering time (Figure 7 and Figure S5). The ABA receptor EjPYL4-like was significantly upregulated on 3 August (T3), especially in 'Baiyu'. At that 
time, both EjPYL4 and EjPYL8 were upregulated in 'Huoju' compared with 'Baiyu' (Figure 8 and Figure S6). EjABI5-like was upregulated in 'Baiyu' throughout bud differentiation (Figure 8 and Figure S6).
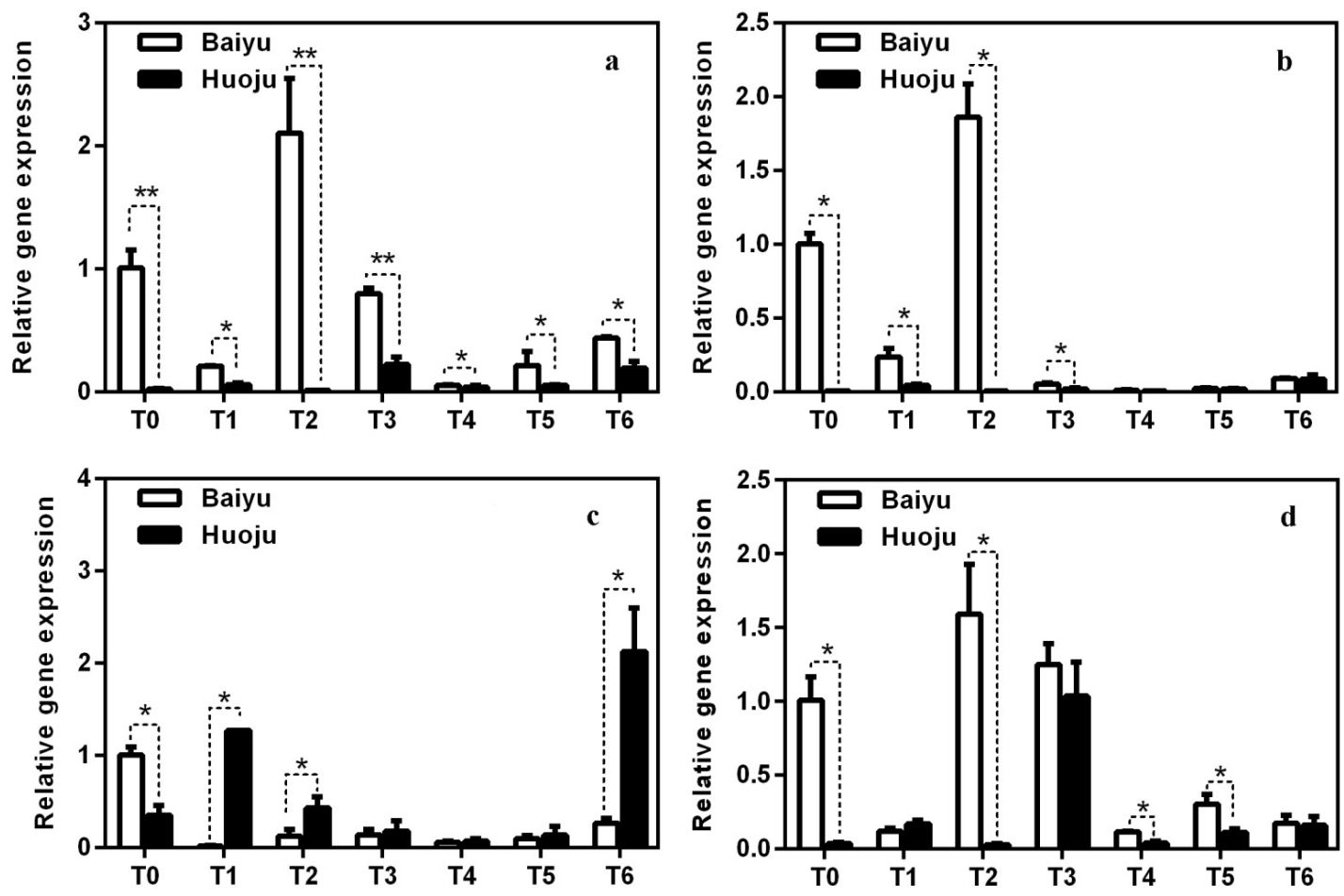

Figure 7. EjABH2 (a), EjABH4 (b), EjABH4-like (c), and EjABH4-like2 (d) expression during loquat bud differentiation. Note: T0-T6 in horizontal axis indicates sampling dates, i.e., 7-13, 7-19, 7-27, 8-03, 8-10, 8-17, and 8-24, respectively; * indicates significance at $p \leq 0.05 ;{ }^{* *}$ indicates significance at $p \leq 0.01$; bars indicate standard error.
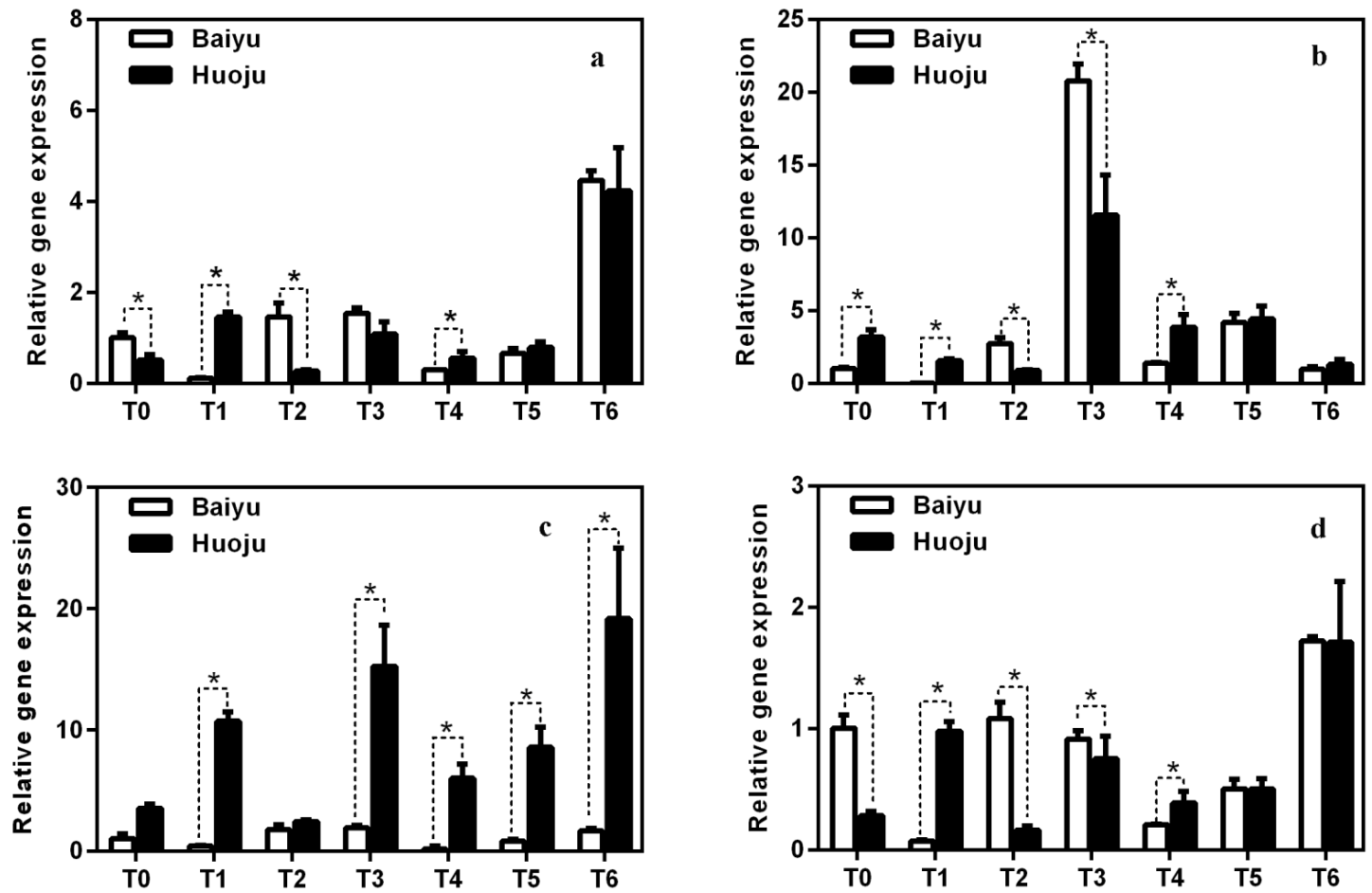

Figure 8. EjPYL4 (a), EjPYL4-like (b), EjPYL8 (c), and EjABI5-like (d) expression during loquat bud differentiation. Note: T0-T6 in horizontal axis indicates sampling dates, i.e., 7-13, 7-19, 7-27, 8-03, 8-10, 8-17, and 8-24, respectively; * indicates significance at $p \leq 0.05$; bars indicate standard error. 


\section{Discussion}

Early flowering is a useful trait in high-value crops as it may enable early fruit harvest and timely market distribution. However, early-flowering loquat cultivars have low yield because they are exposed to low wintertime temperatures. Extension or delay of flowering can reduce the risk of cold stress injury and increase or stabilize production. Nevertheless, flowering time is a complex biological process influenced by numerous factors. Identifying the environmental and genetic mechanisms regulating loquat flowering time could expedite breeding and enhance fruit production (Lin et al. 1999). In the present study, we performed a comparative transcriptome analysis of differentiating loquat flower buds. To the best of our knowledge, this study is the first to use RNA-Seq to identify candidate genes regulating flowering time in loquat.

Transcriptome analysis is an effective approach to study the genes participating in the flowering pathway $[37,38]$. This approach identified DETs associated with various developmental stages of flowering in Moso bamboo [39], apple [40], and chrysanthemum [41-43]. Certain flowering genes such as EjFT1/2 [15], EjAP1 [14], EjSOC1/2 [44], EjGI, and EjCO [45] were recently identified in loquat and cloned from it. However, little is known about the mechanisms regulating the transformation from vegetative to flower bud in loquat. To identify flowering time-related genes and clarify their transcription during bud differentiation in loquat, we conducted a comparative transcriptome analysis on early-flowering 'Baiyu' and late-flowering 'Huoju'. We generated 40.85-46.91 M and 46.26-47.56 M pairs of 150-bp clean reads from 'Baiyu' and 'Huoju', respectively (Tables 1 and 2). After functional annotation, 28,842 DETs were identified (Table 3; Figures 2 and 3; Figures S1 and S2). These data revealed the DET transcription characteristics and biological processes involved in loquat flower bud differentiation.

Several flowering loci have been identified in Arabidopsis. Overexpression of FLOWERING T (FT) leads to early flowering and loss-of-function in the flowering time repressor FLOWERING LOCUS C (FLC). In this manner, the late-flowering phenotype is eliminated [46]. EjLFY-1 in strawberry is homologous to $L E A F Y(L F Y)$ in loquat. Liu et al. reported that $E j L F Y-1$ overexpression accelerates strawberry flowering, shortens the time required for flower induction, and maintains the early-flowering trait in the asexual progeny [47]. Our transcriptome data identified certain known flowering genes such as flowering locus T-like (EjFT-like) [15] and flowering time control protein FY-like (EjFY) [47]. They also revealed other genes possibly regulating the early flowering trait such as flowering locus K (EjFLK), EARLY FLOWERING-LIKE protein (EjELF), CAULIFLOWER A-like (EjCAL1), and others (Figure 4). Our transcriptome data revealed that the expression pattern of unigenes varied significantly between 'Baiyu' and 'Huoju' (Figure S7) and the qRT-PCR indicated that that the flowering-related genes EjFT, EjCAL1-like, EjFY, and EjFLK were upregulated in 'Baiyu' compared with 'Huoju', especially in the early stage of bud differentiation (Figure 5 and Figure S4). Thus, early flowering might have been partially mediated by upregulation of these genes in the buds. However, the functional characteristics and molecular mechanisms of these DETs in the control of loquat flowering time remain to be elucidated.

Flowering is a complex biological process integrating multiple endogenous and environmental signals that ensure timely flowering. This process is regulated by phytohormones $[48,49]$. Several studies demonstrated the importance of interactions among various phytohormones in flower induction [50,51]. In Arabidopsis, ABA is a floral repressor, and flowering is delayed by exogenous ABA application and overexpression of $A B S C I S I C$ ACID INSENSITIVE MUTANT 5 (ABI5). The latter is mediated by upregulation of FLC and the $A B I 5$ promoter encoding bZIP-type transcription factors [52,53]. It was previously reported that $\mathrm{ABA}$ plays the opposite role in loquat, and its level sharply rises during flower bud differentiation [20]. Flowering time was advanced when 200 ppm ABA was sprayed onto loquat apical buds (unpublished data). In the present study, IAA content was low in 'Baiyu' and 'Huoju' apical buds during floral bud development (Figure 1a). Nevertheless, both the ABA content and the ABA:IAA ratio were significantly higher in 
'Baiyu' than 'Huoju' (Figure 1b,c). Hence, the early flowering trait observed in 'Baiyu' may be partially explained by massive ABA accumulation in its buds. The results obtained in the present study supported the theory that in loquat, an increase in the ABA content in the bud is conductive to flower bud formation [20]. We speculated that ABA accumulation during loquat bud differentiation could be promoted by certain environmental conditions such as elevated temperatures and moderate drought. Drought and high temperatures cause water loss, induce stomatal closure, and trigger the drought escape response which, in turn, favors early flowering [54,55]. Flowering induction in the short-day plant Lemna aequinoctialis was associated with high $\mathrm{ABA}$ content [56]. Leng et al. reported that $\mathrm{ABA}$ and its signaling play important roles in regulating floral development and fruit set in sweet cherry. ABA accumulation increased and peaked with floral development. ABA synthetase PaNCED1 expression was consistent with changes in ABA accumulation [57]. In the present study, most ABA signaling genes such as EjABHs, EjPYLs, and EjABI5like showed higher transcription levels in the buds of 'Baiyu' than in those of 'Huoju' (Figures 6-8, Figures S5 and S6). Thus, ABA signaling has a potential regulatory role in loquat flowering time. However, it is unclear whether this positive effect on the expression of genes regulating flowering time can be directly or indirectly attributed to ABA. Furthermore, it is unknown how ABA signaling genes interact with flowering genes and their regulatory networks in loquat. Other stress-related signaling pathways and factors may determine whether flowering will be early or late [58]. Drought stress promoted flowering under long-day conditions in Arabidopsis [59,60] and Citrus latifolia [61]. Salt stress delayed flowering in Arabidopsis [62-64]. Recent research has focused on the potential mechanisms by which stress regulates flowering. Substances produced under stress conditions were considered transmissible flowering stimuli. Salicylic acid was regarded as the most likely compound involved in stress-induced flowering [65]. Nevertheless, the physiological responses and molecular regulatory networks implicated in stress-induced loquat flowering remain obscure. In addition, flowering is influenced by multiple factors such as tree age and nutrition, phytohormones, sunshine duration, ambient temperature, and other environmental conditions. A great number of transcription factors (TFs) have been identified, of which $A P 2, A R F, A R R-B, C 2 H 2, E R F, M Y B$ family, WORK, $b H L H$, and $b Z I P$ were the predominated subgroups (Figure S8, Tables S9-S15), these TFs may participate in the loquat flowering pathway. However, functional roles of these DETs and TFs associated with these pathways have yet to be identified.

\section{Conclusions}

To the best of our knowledge, this report is the first to provide comprehensive transcriptome and DET profiling data associated with loquat floral bud differentiation. Approximately 28,842 DETs involved in multiple regulatory pathways were significantly differentially regulated between early-flowering and late-flowering loquat cultivars. Fortytwo candidate DETs were identified that might play important roles in flowering time control. Seventeen candidate DETs involved in the ABA signaling pathway were identified. The results of the present study could help clarify the gene transcription and regulatory networks involved in loquat flowering time. They also provide an empirical basis for further study on the progression of bud development and the determination of flowering time in loquat.

Supplementary Materials: Supplementary Materials are available online at https://www.mdpi. com/article/10.3390/horticulturae7070171/s1. Figure S1: Sequence length distributions of unigenes from fully assembled transcriptome during loquat flower bud differentiation. Figure S2: Distribution of top ten species of loquat unigenes in NR database. Figure S3: Volcano graph of up-/downregulated unigenes in each library. Figure S4. Fpkm-based expression of EjFT (a), EjFY (b), EjFLK (c) and EjCAL1-like (d) during bud differentiation in loquat. Note: T0-T6 in horizontal axis indicates sampling dates, i.e., 7-13, 7-19, 7-27, 8-03, 8-10, 8-17, and 8-24, respectively. Figure S5: Fpkmbased expression of EjABH2 (a), EjABH4 (b), EjABH4-like (c), and EjABH4-like2 (d) during bud differentiation in loquat. Note: T0-T6 in horizontal axis indicates sampling dates, i.e., 7-13, 7-19, 
7-27, 8-03, 8-10, 8-17, and 8-24, respectively. Figure S6: Fpkm-based expression of EjPYL4 (a), EjPYL4-like (b), EjPYL8 (c), and EjABI5-like (d) during bud differentiation in loquat. Note: T0-T6 in horizontal axis indicates sampling dates, i.e., $7-13,7-19,7-27,8-03,8-10,8-17$, and 8-24, respectively. Figure S7: The gene expression differences between 'Baiyu' and 'Huoju'. Figure S8: Transcription factors distribution in loquat. Figure S9: GO enrichment of Huoju_713-vs-Baiyu_713. Figure S10: GO enrichment of Huoju_719-vs-Baiyu_719. Figure S11: GO enrichment of Huoju_727-vs-Baiyu_727. Figure S12: GO enrichment of Huoju_803-vs-Baiyu_803. Figure S13: GO enrichment of Huoju_810vs-Baiyu_810. Figure S14: GO enrichment of Huoju_817-vs-Baiyu_817. Figure S15: GO enrichment of Huoju_824-vs-Baiyu_824. Table S1: Linear regression of ABA and IAA measured by LC-MS. Table S2: Primers for qRT-PCR analysis used in this study. Table S3: Matching of unigenes obtained by RNA-Seq data against genome database of loquat. Table S4: Unigene annotation based on GO enrichment. Table S5: Unigene annotation based on KEGG enrichment. Table S6: List of all DETs information with unigene ID, functional annotation and FPKM value. Table S7: The 42 candidate unigenes involved in flowering. Table S8: The 23 candidate unigenes involved in ABA signaling. Table S9: Transcription factors distribution in Huoju_713-vs-Baiyu_713. Table S10: Transcription factors distribution in Huoju_719-vs-Baiyu_719. Table S11: Transcription factors distribution in Huoju_727-vs-Baiyu_727. Table S12: Transcription factors distribution in Huoju_803-vs-Baiyu_803. Table S13: Transcription factors distribution in Huoju_810-vs-Baiyu_810. Table S14: Transcription factors distribution in Huoju_817-vs-Baiyu_817. Table S15: Transcription factors distribution in Huoju_824-vs-Baiyu_824.

Author Contributions: Conceptualization, H.A. and X.Z.; methodology, S.J.; validation, H.A., S.J. and X.Z.; formal analysis, H.A. and J.Z.; investigation, F.X., J.Z. and X.Z.; resources, X.Z.; data curation, H.A. and S.J.; writing-original draft preparation, H.A.; writing-review and editing, S.J. and X.Z.; visualization, H.A.; supervision, X.Z. All authors have read and agreed to the published version of the manuscript.

Funding: This work was supported by the Agriculture Applied Technology Development Program of the Shanghai Agriculture Committee (No. 2018-1-7).

Data Availability Statement: All raw data were deposited in the Genome Sequence Archive (GSA) at the National Genomics Data Centre under Project Nos. CRR121350-CRR121363.

Acknowledgments: The authors thank Ouyi Biotech Co. Ltd. (Shanghai, China) for their technical support with the transcriptome analysis.

Conflicts of Interest: The authors declare that they have no competing interest.

\section{References}

1. Zhao, W.; Gu, R.; Che, G.; Cheng, Z.; Zhang, X. CsTFL1b may regulate the flowering time and inflorescence architecture in cucumber (Cucumis satious L.). Biochem. Biophys. Res. Commun. 2018, 499, 307-313. [CrossRef] [PubMed]

2. Yano, M.; Kojima, S.; Takahashi, Y.; Lin, H.; Sasaki, T. Genetic control of flowering in rice, a short-day Plant. Plant Physiol. 2001, 127, 1425-1429. [CrossRef]

3. Zhang, J.Z.; Ai, X.Y.; Sun, L.M.; Zhang, D.L.; Guo, W.W.; Deng, X.X.; Hu, C.G. Transcriptome profile analysis of flowering molecular processes of early flowering trifoliate orange mutant and the wild type [Poncirus trifoliata (L.) Raf.] by massively parallel signature sequencing. BMC Genom. 2011, 12, 63. [CrossRef] [PubMed]

4. Amasino, R. Seasonal and developmental timing of flowering. Plant J. 2010, 61, 1001-1013. [CrossRef] [PubMed]

5. Srikanth, A.; Schmid, M. Regulation of flowering time: All roads leads to Rome. Cell Mol. Life Sci. 2011, 68, 2013-2037. [CrossRef] [PubMed]

6. Andres, F.; Coupland, G. The genetic basis of flowering responses to seasonal cues. Nat. Rev. Genet. 2012, 13, 627. [CrossRef]

7. Wils, C.R.; Kaufmann, K. Gene-regulatory networks controlling inflorescence and flower development in Arabidopsis thaliana. Biochim. Biophys. Acta Gene Regul. Mech. 2017, 1860, 95-105. [CrossRef]

8. Fornara, F.; de Montaigu, A.; Coupland, G. SnapShot: Control of flowering in Arabidopsis. Cell 2010, 141, 550.e1. [CrossRef]

9. Li, C.; Lin, H.; Dubcovsky, J. Factorial combinations of protein interactions generate a multiplicity of florigen activation complexes in wheat and barley. Plant J. 2015, 84, 70-82. [CrossRef]

10. Hoenicka, H. Over-expression of an FT-homologous gene of apple induces early flowering in annual and perennial plants. Planta 2010, 223, 1309-1324.

11. Foster, T.M.; Watson, A.E.; Hooijdonk, B.M.V.; Schaffer, R.J. Key flowering genes including FT-like genes are upregulated in the vasculature of apple dwarfing rootstocks. Tree Genet. Genom. 2014, 10, 189-202. [CrossRef] 
12. Ito, A.; Saito, T.; Nishijima, T.; Moriguchi, T. Effect of extending the photoperiod with low-intensity red or far-red light on the timing of shoot elongation and flower-bud formation of 1-year-old Japanese pear (Pyrus pyrifolia). Tree Physiol. 2014, 34, 534-546. [CrossRef]

13. Chen, Y.; Jiang, P.; Thammannagoewda, S.; Liang, H.; Wilde, H.D. Characterization of peach TFL1 and comparison with FT/TFL1 gene families of Rosaceae. J. Am. Soc. Hort. Sci. 2013, 138, 12-17. [CrossRef]

14. Liu, Y.; Song, H.; Liu, Z.; Hu, G.; Lin, S. Molecular characterization of loquat EjAP1 gene in relation to flowering. Plant Growth Regul. 2013, 70, 287-296. [CrossRef]

15. Reig, C.; Gil-Munoz, F.; Vera-Sirera, F.; Garcia-Lorca, A.; Martinez-Fuentes, A.; Mesejo, C.; Perez-Amador, M.A.; Agusti, M. Bud sprouting and floral induction and expression of FT in loquat [Eriobotrya japonica (Thunb.) Lindl.]. Planta 2017, 246, 915-925. [CrossRef]

16. Jing, D.; Chen, W.; Hu, R.; Zhang, Y.; Xia, Y.; Wang, S.; He, Q.; Guo, Q.; Liang, G. An integrative analysis of transcriptome, proteome and hormones reveals key differentially expressed genes and metabolic pathways involved in flower development in loquat. Int. J. Mol. Sci. 2020, 21, 5107. [CrossRef] [PubMed]

17. Cheng, Y.; Zhao, Y. A role for auxin in flower development. J. Integr. Plant Biol. 2007, 49, 99-104. [CrossRef]

18. Matsoukas, I.G. Interplay between sugar and hormone signaling pathways modulate floral signal transduction. Front. Genet. 2014, 5, 218. [CrossRef] [PubMed]

19. Shu, K.; Chen, Q.; Wu, Y.; Liu, R.; Zhang, H.; Wang, S.; Tang, S.; Yang, W.; Xie, Q. ABSCISIC-ACID-INSENSITIVE 4 negatively regulates flowering through directly promoting Arabidopsis FLOWERING LOCUS C transcription. J. Exp. Bot. 2016, 67, 195-205. [CrossRef]

20. Liu, Z.; Lin, S.; Chen, H. Time course changes of endogenous hormone levels during the floral and vegetative bud formation in loquat (Eriobotrya japonica Lindl.). Acta Horti. Sinica. 2007, 34, 339-344. (In Chinese)

21. Fernandez, M.D.; Hueso, J.J.; Cuevas, J. Water stress integral for successful modification of flowering dates in 'Algerie' loquat. Irrig. Sci. 2010, 28, 127-134. [CrossRef]

22. Lin, S.Q.; Sharpe, R.H.; Janick, J. Loquat: Botany and horticulture. Hortic. Rev. 1999, 23, 233-276.

23. Demirkeser, T.H.; Caliskan, O.; Polat, A.A.; Ozgen, M.; Serce, S. Effect of natural lipid on pollen germination and pollen tube growth o loquat. Asian J. Plant Sci. 2007, 6, 304-307. [CrossRef]

24. Jiang, S.; Luo, J.; Xu, F.; Zhang, X. Transcriptome analysis reveals candidate genes involved in gibberellins-induced fruit setting in tripod loquat (Eriobotrya japonica). Front. Plant Sci. 2016, 7, 1924. [CrossRef]

25. Song, H.; Zhao, X.; Hu, W.; Wang, X.; Shen, T.; Yang, L. Comparative transcriptional analysis of loquat fruit identifies major signal networks involved in fruit development and ripening process. Int. J. Mol. Sci. 2016, 17, 1837. [CrossRef] [PubMed]

26. Gong, R.G.; Lai, J.; Liao, M.A.; Wang, Z.H.; Liang, G.L. Analysis of alterations to the transcriptome of loquat (Eriobotrya japonica Lindl.) under low temperature stress via de novo sequencing. Genet. Mol. Res. 2015, 14, 9423-9436. [CrossRef] [PubMed]

27. Xu, H.; Li, X.; Chen, J. Comparative transcriptome profiling of freezing stress responses in loquat (Eriobotrya japonica) fruitlets. $J$. Plant Res. 2017, 130, 893-907. [CrossRef] [PubMed]

28. Niu, Q.; Zong, Y.; Qian, M.; Yang, F.; Teng, Y. Simultaneous quantitative determination of major plant hormones in pear flowers and fruit by UPLC/ESI-MS/MS. Anal. Method UK 2014, 6, 1766. [CrossRef]

29. Bolger, A.M.; Lohse, M.; Usadel, B. Trimmomatic: A flexible trimmer for Illumina sequence data. Bioinformatics 2014, 30, 2114-2120. [CrossRef]

30. Grabherr, M.G.; Haas, B.J.; Yassour, M.; Levin, J.Z.; Thompson, D.A.; Amit, I.; Adiconis, X.; Fan, L.; Raychowdhury, R.; Zeng, Q.; et al. Trinity: Reconstructing a full-length transcriptome without genome from RNA_Seq data. Nat. Biotechnol. $2011,2,644-652$. [CrossRef]

31. Altschul, S.F.; Gish, W.; Miller, W.; Myers, E.W.; Lipman, D.J. Basic local alignment search tool. J. Mol. Biol. 1990, 215, 403-410. [CrossRef]

32. Kanehisa, M.; Araki, M.; Goto, S.; Hattori, M.; Hirakawa, M.; Itoh, M.; Katayama, T.; Kawashima, S.; Okuda, S.; Tokimatsu, T.; et al. KEGG for linking genomes to life and the environment. Nucleic Acids Res. 2008, 36, D480-D484. [CrossRef] [PubMed]

33. Trapnell, C.; Williams, B.A.; Pertea, G.; Mortazavi, A.; Kwan, G.; van Baren, M.J.; Salzberg, S.L.; Wold, B.J.; Pachter, L. Transcript assembly and quantification by RNA_seq reveals unannotated transcripts and isoform switching during cell differentiation. Nat. Biotechnol. 2010, 28, 511-515. [CrossRef]

34. Langmead, B.; Salzberg, S.L. Fast gapped-read alignment with Bowtie 2. Nat. Methods 2012, 9, 357-359. [CrossRef]

35. Robert, A.; Pachter, L. Streaming fragment assignment for real-time analysis of sequencing experiments. Nat. Methods 2013 10, 71-73. [CrossRef]

36. Anders, S.; Huber, W. Differential expression of RNA-Seq data at the gene level sequencing experiment. EMBL 2013, $10, \mathrm{f} 10000$.

37. Zhang, J.; Wu, K.; Zeng, S.; da Silva, J.A.T.; Zhao, X.; Tian, C.E.; Xia, H.; Duan, J. Transcriptome analysis of Cymbidium sinense and its application to the identification of genes associated with floral development. BMC Genom. 2013, 14, 279. [CrossRef]

38. Wen, Z.; Guo, W.; Li, J.; Lin, H.; He, C.; Liu, Y.; Zhang, Q.; Liu, W. Comparative transcriptomic analysis of vernalization- and cytokinin-induced floral transition in Dendrobium nobile. Sci. Rep. 2017, 7, 45748. [CrossRef] [PubMed]

39. Gao, J.; Zhang, Y.; Zhang, C.; Qi, F.; Li, X.; Mu, S.; Peng, Z. Characterization of the floral transcriptome of Moso bamboo (Phyllostachys edulis) at different flowering developmental stages by transcriptome sequencing and RNA-seq analysis. PLoS ONE 2014, 9, e98910. [CrossRef] 
40. Li, Y.; Zhang, D.; Zhang, X.; Xing, L.; Fan, S.; Ma, J.; Zhao, C.; Du, L.; Han, M. A transcriptome analysis of two apple (Malus a domestica) cultivars with different flowering abilities reveals a gene network module associated with floral transitions. Sci. Hortic. 2018, 239, 269-281. [CrossRef]

41. Liu, H.; Sun, M.; Du, D.; Pan, H.; Cheng, T.; Wang, J. Whole-transcriptome analysis of differentially expressed genes in the vegetative buds, floral buds, and buds of Chrysanthemum morifolium. PLoS ONE 2015, 10, e0128009. [CrossRef]

42. Ren, L.; Liu, T.; Cheng, Y.; Sun, J.; Gao, J.; Dong, B.; Chen, S.; Chen, F.; Jiang, J. Transcriptomic analysis of differentially expressed genes in the floral transition of the summer flowering chrysanthemum. BMC Genom. 2016, 17, 673. [CrossRef] [PubMed]

43. Cheng, P.; Dong, B.; Wang, H.; Cao, P.; Liu, T.; Liu, Y.; Gao, J.; Liao, Y.; Fang, W.; Chen, S.; et al. A transcriptomic analysis targeting genes involved in the floral transition of winter-flowering chrysanthemum. J. Plant Growth Regul. 2018, 37, 220-232. [CrossRef]

44. Jiang, Y.; Peng, J.; Zhu, Y.; Su, W.; Zhang, L.; Jing, Y.; Lin, S.; Gao, Y. The role of EjSOC1s in flower initiation in Eriobotrya japonica. Front. Plant Sci. 2019, 10, 253. [CrossRef]

45. Zhang, L.; Jiang, Y.; Zhu, Y.; Su, W.; Long, T.; Huang, T.; Peng, J.; Yu, H.; Lin, S.; Gao, Y. Functional characterization of GI and CO homologs from Eriobotrya deflexa Nakai forma koshunensis. Plant Cell Rep. 2019, 38, 533-543. [CrossRef]

46. Michaels, S.D.; Amasino, R.M. Loss of FLOWERING LOCUS C activity eliminates the late-flowering phenotype of FRIGIDA and autonomous pathway mutations but not responsiveness to vernalization. Plant Cell 2001, 13, 934-941. [CrossRef]

47. Liu, Y.; Zhao, Q.; Meng, N.; Song, H.; Li, C.; Hu, G.; Wu, J.; Lin, S.; Zhang, Z. Over-expression of EjLFY-1 leads to an early flowering habit in strawberry (Fragaria $\times$ ananassa) and its asexual progeny. Front. Plant Sci. 2017, 8, 496. [PubMed]

48. Mouradov, A.; Cremer, F.; Coupland, G. Control of flowering time: Interacting pathways as a basis for diversity. Plant Cell 2002 14, S111-S130. [CrossRef]

49. Campos-Rivero, G.; Osorio-Montalvo, P.; Sanchez-Borges, R.; Us-Camas, R.; Duarte-Ake, F.; De-la-Pena, C. Plant hormone signaling in flowering: An epigenetic point of view. J. Plant Physiol. 2017, 214, 16-27. [CrossRef]

50. Domagalska, M.A.; Sarnowska, E.; Nagy, F.; Davis, S.J. Genetic analysis of interactions among gibberellins, abscisic acid and brassinosteroids in the control of flowering time in Arabidopsis thaliana. PLoS ONE 2010, 5, e14012. [CrossRef] [PubMed]

51. Frankowski, K.; Wilmowicz, E.; Kucko, A.; Kesy, J.; Swiezawska, B.; Kopcewicz, J. Ethylene, auxin, and abscisic acid interactions in the control of photoperiodic flower induction in Pharbitis nil. Biol. Plantar. 2014, 58, 305-310. [CrossRef]

52. Brocard, I.M.; Lynch, T.J.; Finkelstein, R.R. Regulation and role of the Arabidopsis abscisic acid-insensitive 5 in abscisic acid, sugar, and stress response. Plant Physiol. 2002, 129, 1533-1543. [CrossRef]

53. Wang, Y.; Li, L.; Ye, T.; Chen, X.; Wu, Y. The inhibitory effect of ABA on floral transition is mediated by ABI5 in Arabidopsis. J. Exp. Bot. 2013, 64, 675-684. [CrossRef]

54. Sherrard, M.E.; Maherali, H. The adaptive significance of drought escape in Avena barbata, an annual grass. Evolution 2006, 60, 2478-2489. [CrossRef]

55. Su, Z.; Ma, X.; Guo, H.; Sukiran, N.L.; Guo, B.; Assmann, S.M.; Ma, H. Flower development under drought stress: Morphological and transcriptomic analyses reveal acute responses and long-term acclimation in Arabidopsis. Plant Cell 2013, 25, $3785-3807$. [CrossRef]

56. Tsao, T.H.; Wang, H.W.; Jiao, J.P.; Tan, Z.Y. Changes in endogenous ABA and GA contents during floral induction of Lemna aequinoctialis. Act. Botan. Neeriand. 1986, 35, 443-448. [CrossRef]

57. Leng, P.; Zhang, Y.; Du, Y.; Wang, J.; Jiang, L.; Kai, W.; Liang, B.; Zhai, X.; Sun, Y.; Liu, H.; et al. Expression pattern of ABA metabolic and signaling genes during floral development and fruit set in sweet cherry. Plant Growth Regul. 2018, 84, 71-80. [CrossRef]

58. Takeno, K. Stress-induced flowering: The third category of flowering response. J. Exp. Bot. 2016, 67, 4925-4934. [CrossRef] [PubMed]

59. Riboni, M.; Galbiati, M.; Tonelli, C.; Conti, L. GIGANTEA enables drought escape response via ABA-dependent activation of the florigens and SUPPERSSOR OF OVEREXPRESSION OF CONSTANS1. Plant Physiol. 2013, 162, 1706-1719. [CrossRef]

60. Riboni, M.; Test, A.R.; Galbiati, M.; Tonelli, C.; Conti, L. Environmental stress and flowering time. Plant Signal. Behav. 2014, 9, e29036. [CrossRef]

61. Southwick, S.M.; Davenport, T.L. Characterization of water stress and low temperature effects on flower induction in Citrus. Plant Physiol. 1986, 81, 26-29. [CrossRef]

62. Kim, S.G.; Kim, S.Y.; Park, C.M. A membrane-associated NAC transcription factor regulates salt-responsive flowering via FLOWERING LOCUS T in Arabidopsis. Planta 2007, 226, 647-654. [CrossRef]

63. Ryu, J.Y.; Lee, H.J.; Seo, P.J.; Jung, J.H.; Ahn, J.H.; Park, C.M. The Arabidopsis floral repressor BFT delays flowering by competing with FT for FD binding under high salinity. Mol. Plant 2013, 7, 377-387. [CrossRef]

64. Li, K.; Wang, Y.; Han, C.; Zhang, W.; Jia, H.; Li, X. GA signaling and CO/FT regulatory module mediate salt-induced late flowering in Arabidopsis thaliana. Plant Growth Regul. 2007, 53, 195-206. [CrossRef]

65. Kohli, A.; Sreenivasulu, N.; Lakshmanan, P.; Kumar, P.P. The phytohormone crosstalk paradigm takes center stage in understanding how plants respond to abiotic stresses. Plant Cell Rep. 2013, 32, 945-957. [CrossRef] [PubMed] 